\title{
Optimal use of lenvatinib in the treatment of advanced thyroid cancer
}

\author{
Shunji Takahashi ${ }^{*}$, Naomi Kiyota ${ }^{2}$ and Makoto Tahara ${ }^{3}$
}

\begin{abstract}
The development of orally active, multitargeted kinase inhibitors (MKIs) represents a significant advance in the treatment of progressive, metastatic thyroid cancer. Lenvatinib, an MKI targeting vascular endothelial growth factor receptor, fibroblast growth factor receptor, platelet-derived growth factor receptor, c-Kit, and RET, has shown efficacy in stabilizing previously progressive disease, with emerging evidence of a possible benefit in terms of overall survival. However, lenvatinib is associated with a side-effect profile similar to those of other MKIs that might affect the outcome of therapy.

The aim of this review is to summarize the clinical efficacy and safety of MKIs in the treatment of advanced thyroid cancer in pivotal phase III trials. Common adverse events that may occur during lenvatinib therapy and their management are discussed, including conditions in which its administration should be temporarily withdrawn and resumed pending resolution of adverse events. We focus on data from a subanalysis of Japanese patients in the SELECT trial and in a post-marketing study in Japan.

We suggest that lenvatinib is a valuable treatment option for advanced differentiated thyroid cancer. Monitoring and careful management of adverse events including supportive care are required to ensure continuation of therapy.
\end{abstract}

Keywords: Adverse event, Lenvatinib, Multitargeted kinase inhibitor, Thyroid cancer, Vascular endothelial growth factor receptor

\section{Background}

Thyroid cancer is a common endocrine malignancy that can be divided into three primary histologic types (differentiated, medullary, and anaplastic). These histologic subtypes have different characteristics and prognoses. The initial treatment of differentiated thyroid cancer is surgical resection, thyroid hormone therapy or radioactive iodine in selected cases. Radioactive iodine can be curative in some cases of metastatic disease and thyroidstimulating hormone-suppressive hormone therapy can prevent disease progression. However, until recently, the treatment options were limited for patients with metastatic disease that did not respond to the above treatments and for those with medullary and anaplastic thyroid cancer.

\footnotetext{
* Correspondence: s.takahshi-chemotherapy@jfcr.or.jp

'Department of Medical Oncology, Cancer Institute Hospital, Japanese Foundation for Cancer Research, 3-8-31 Ariake, Koto-ku, Tokyo 135-8550, Japan

Full list of author information is available at the end of the article
}

Several oral multi-targeted kinase inhibitors (MKIs) have now been approved in Japan and other countries for advanced thyroid cancer, based on the results of phase III trials [1-3]. In Japan, sorafenib and lenvatinib are approved for unresectable differentiated as well as medullary and anaplastic thyroid cancer. Vandetanib is approved for unresectable medullary thyroid carcinoma. Cabozantinib is also approved for medullary thyroid cancer in Western countries, but not yet in Japan. These MKIs target the vascular endothelial growth factor receptor (VEGFR) but their distinct kinase inhibition profiles may result in differences in efficacy and adverse events.

Preclinical cancer models have demonstrated that lenvatinib targets VEGFR 1-3, fibroblast growth factor receptors (FGFR) 1-4, platelet-derived growth factor receptor $\alpha$, c-Kit, and RET $[4,5]$. By targeting the VEGF pathway in particular, it exerts antitumor activity by inhibiting angiogenesis. Lenvatinib and other MKIs represent a significant breakthrough in the treatment of 
advanced thyroid cancer, but the timing of MKI therapy and the selection of individual agents are not well established, given the absence of direct comparisons of the efficacy and safety of MKIs [6].

MKIs have unique safety profiles and significant toxicities may occur. Therefore, a thorough understanding of the adverse events associated with lenvatinib is needed, along with a comprehensive assessment of adverse events and their management, including the need for drug interruption and dose reduction, and potential treatment discontinuation, during lenvatinib therapy. In this review, we describe the efficacy and safety of lenvatinib in the treatment of advanced thyroid cancer based on data from global and Japanese clinical trials, focusing on the management of common adverse events associated with lenvatinib. The optimal use of lenvatinib through the appropriate management of adverse events will also be discussed.

\section{Clinical efficacy}

The efficacy of lenvatinib in major trials is summarized in Table 1. A global, randomized, double-blind, phase III study (SELECT, Study of E7080 Lenvatinib in Differentiated Cancer of the Thyroid) was conducted to compare the effects of lenvatinib $(n=261)$ versus placebo $(n=131)$ in patients with radioiodine-refractory, differentiated thyroid cancer, including those who had no or one prior treatment with a tyrosine kinase inhibitor [2]. The median progression-free survival (18.3 versus 3.6 months; $p<0.001)$ and the overall response rate (64.8\% versus $1.5 \% ; p<0.001)$ were significantly better in the lenvatinib group than in the placebo group.

In a subgroup analysis of Japanese patients (lenvatinib, $n=30$; placebo, $n=10$ ) in the SELECT trial [7], the median progression-free survival was longer in the lenvatinib group (16.5 months versus 3.7 months; $p=0.067$ ) but statistical significance was not reached, presumably because of the relatively small sample size. The overall response rate was greater in the lenvatinib group (63.3\% versus $0 \% ; p=0.0004)$. The subset was not a randomized group and the $p$-value is nominal.

Two phase II studies examined the effects of lenvatinib in patients with either advanced radioiodine-refractory, differentiated or medullary thyroid carcinomas in Western countries, which included those who had received prior chemotherapy or tyrosine kinase inhibitor therapy $[8,9]$. The median progression-free survival times in patients with differentiated or medullary thyroid carcinomas were 12.6 months and 9.0 months, while the overall response rates were $50 \%$ and $36 \%$, and the disease control rates were $93 \%$ and $80 \%$, respectively.

Another phase II study examined the effects of lenvatinib in 51 Japanese patients with either unresectable radioiodine-refractory, differentiated, medullary, or anaplastic thyroid carcinomas [10, 11]. Previous use of sorafenib for differentiated thyroid cancer was allowed. The median progression-free survival times in patients with differentiated, medullary, or anaplastic thyroid carcinomas were 25.8 months, 9.2 months and 7.4 months, and the median overall survival times were 31.8 months, 12.1 months, and 10.6 months, respectively. The overall response rates were $68 \%, 22 \%$, and $24 \%$, and the disease control rates were $100 \%, 100 \%$, and $94 \%$, respectively. These data suggest that lenvatinib may induce tumor shrinkage and prevents disease progression in Japanese patients with medullary or anaplastic thyroid cancer in addition to advanced, differentiated thyroid cancer. Currently, lenvatinib has not been approved for medullary or anaplastic thyroid carcinomas outside Japan and clinical studies are underway in Western countries (NCT00784303, NCT02657369).

Sorafenib has also demonstrated benefit in patients with radioiodine-refractory differentiated thyroid cancer with locally advanced or metastatic disease. In a multicenter, double-blind, phase III trial (DECISION) involving 417 patients randomized to sorafenib or placebo

Table 1 Summary of efficacy in clinical trials of lenvatinib in the treatment of thyroid cancer

\begin{tabular}{|c|c|c|c|c|c|c|c|}
\hline Study [ref] & Arm & Subtype & N & $\begin{array}{l}\text { PFS, months } \\
(95 \% \mathrm{Cl})\end{array}$ & $\begin{array}{l}\text { OS, months } \\
(95 \% \mathrm{Cl})\end{array}$ & $\begin{array}{l}\text { ORR } \\
(\%)\end{array}$ & $\begin{array}{l}\text { DCR } \\
(\%) \\
\end{array}$ \\
\hline Phase II [8] & Lenvatinib & DTC & 58 & $12.6(9.9-16.1)$ & - & 50 & 93 \\
\hline Phase II [9] & Lenvatinib & MTC & 59 & 9.0 (7.0-NE) & - & 36 & 80 \\
\hline \multirow[t]{3}{*}{ Phase II (Japan) [10] } & \multirow[t]{3}{*}{ Lenvatinib } & DTC & 25 & 25.8 (18.4-NE) & 31.8 (31.8-NE) & 68 & 100 \\
\hline & & MTC & 9 & $9.2(1.8-\mathrm{NE})$ & 12.1 (3.8-NE) & 22 & 100 \\
\hline & & ATC & 17 & $7.4(1.7-12.9)$ & $10.6(3.8-19.8)$ & 24 & 94 \\
\hline \multirow[t]{2}{*}{ Phase III (general population) [2] } & Lenvatinib & DTC & 261 & 18.3 (15.1-NE) & $\mathrm{NE}$ & 64.8 & 87.7 \\
\hline & Placebo & & 131 & $3.6(2.2-3.7)$ & & 1.5 & 55.7 \\
\hline \multirow[t]{2}{*}{ Phase III (Japanese population) [7] } & Lenvatinib & DTC & 30 & 16.5 (7.4-NE) & NE & 63.3 & 90.0 \\
\hline & Placebo & & 10 & $3.7(1.6-9.1)$ & & 0.0 & 60.0 \\
\hline
\end{tabular}

ATC anaplastic thyroid cancer; DCR disease control rate; DTC differentiated thyroid cancer; MTC medullary thyroid cancer; NE, not estimable; ORR overall response rate; OS overall survival; PFS progression-free survival 
who had not previously received tyrosine kinase inhibitor therapy, the primary endpoint of median progression-free survival was 10.8 months versus 5.8 months (HR 0.59, 95\% CI 0.45-0.76), respectively [1]. For advanced medullary thyroid cancer, vandetanib was effective in a phase III trial of 331 patients by significantly extending progression-free survival (HR 0.46, 95\% CI 0.31-0.69) [3]. Although the median progression-free survival was not reached, it was predicted to be 30.5 months in the vandetanib group versus 19.3 months in the placebo group. A double-blind phase III trial also demonstrated a benefit of cabozantinib in patients with progressive, metastatic medullary thyroid cancer, with a median progressionfree survival of 11.2 months versus 4 months for placebo [12].

\section{Type and management of adverse events during lenvatinib therapy}

Various adverse events have been observed during lenvatinib therapy, which are similar to those reported for other MKIs targeting the VEGF signaling pathway $[2,7]$. The most common adverse events include hypertension, diarrhea, fatigue, palmar-plantar erythrodysesthesia and proteinuria (Table 2). In this section, we discuss the characteristics of adverse events that may arise during lenvatinib therapy. We also discuss possible strategies to manage these adverse events, including dose reduction, treatment interruption, supportive medications, and treatment discontinuation, which would mirror those used for other MKIs [13]. A starting dose of $24 \mathrm{mg}$ once a day is indicated for lenvatinib treatment and, in the case of an intolerable adverse event, stepwise dose reductions are recommended from $24 \mathrm{mg}$ to $20 \mathrm{mg}, 14 \mathrm{mg}$, and $10 \mathrm{mg}$, as recommended in phase 2 and 3 studies of lenvatinib [2, 7]. In the protocols of these two trials, a reduction in dose to $8 \mathrm{mg}$ or $4 \mathrm{mg}$ was requested when a patient needed the dose to be reduced to $<10 \mathrm{mg}$. Resumption of lenvatinib at the reduced dose is usually recommended after interruption, but if the dose at the time of interruption was $<14 \mathrm{mg}$, resumption of the same dose should be considered in order to maintain the dose intensity.
The adverse events are described in decreasing order of frequency (any grade) in the SELECT trial [2], with a particular focus on those that occurred in Japanese patients.

\section{Hypertension}

Because VEGF influences vascular resistance through the production of nitric oxide and enhancing angiogenesis, hypertension frequently occurs with VEGF inhibitors, including sorafenib, vandetanib, cabozantinib, and lenvatinib $[14,15]$. Hypertension more frequently occurred in Japanese patients in the SELECT trial [7]. Hypertension of any grade occurred in $86.7 \%$ of Japanese patients and grade $\geq 3$ occurred in $80.0 \%$ (Table 2), whereas the occurrence of hypertension in the general population was $67.8 \%$ for any grade and $41.8 \%$ for grade $\geq 3$ [2]. The overall incidence of hypertension in lenvatinib-treated patients was somewhat higher than that reported for sorafenib in the DECISION trial $(40.6 \%$ for any grade; $9.7 \%$ for grade $\geq 3$ ) [1]. The onset of hypertension was relatively early in the SELECT trial, with a median time to onset of 2.3 weeks [16], while it was 8 days in Japanese patients (unpublished data). Interestingly, treatment-emergent hypertension was associated with a 5.9-month median progression-free survival advantage (hazard ratio 0.59 [95\% CI 0.39-0.88]; $P=0.009)$. Given the early onset and high rate of development of hypertension, the baseline blood pressure should be $<140 / 90 \mathrm{mmHg}$ and blood pressure should be measured regularly at home and at each clinical visit during the first few weeks of treatment, at least. In the SELECT study, patients with confirmed systolic BP $\geq 140 \mathrm{mmHg}$ or diastolic BP $\geq 90 \mathrm{mmHg}$ were administered antihypertensive agents and monitored every 2 weeks. Patients with systolic BP $\geq 160 \mathrm{mmHg}$ or diastolic BP $\geq 100 \mathrm{mmHg}$, despite optimal management, had their treatment dose reduced.

We propose a management plan for hypertension during lenvatinib therapy (Fig. 1), according to the severity of hypertension. Patients should measure blood pressure at the same time in the morning once a day at home. When an elevated blood pressure is detected, the blood pressure should be re-measured after an interval of $5 \mathrm{~min}$ or longer. If the blood pressure remains elevated

Table 2 Major adverse events associated with lenvatinib

\begin{tabular}{llllllll}
\hline & & Hypertension & Diarrhea & Fatigue & PPE & Proteinuria & Thrombocytopenia \\
\hline Phase III (general population) ( $n=261)$ [2] & Any grade & 68 & 59 & 59 & 32 & 31 & 9 \\
& Grade $\geq 3$ & 42 & 8 & 9 & 3 & 10 & 2 \\
Phase III (Japanese population) ( $n=30)$ [7] & Any grade & 87 & 60 & 60 & 70 & 63 & 47 \\
& Grade $\geq 3$ & 80 & 0 & 13 & 3 & 20 & 7 \\
\hline
\end{tabular}

Events are listed in order of the most frequent events of any grade in the general population

Data are shown as incidence (\%). PPE, palmar-plantar erythrodysesthesia 


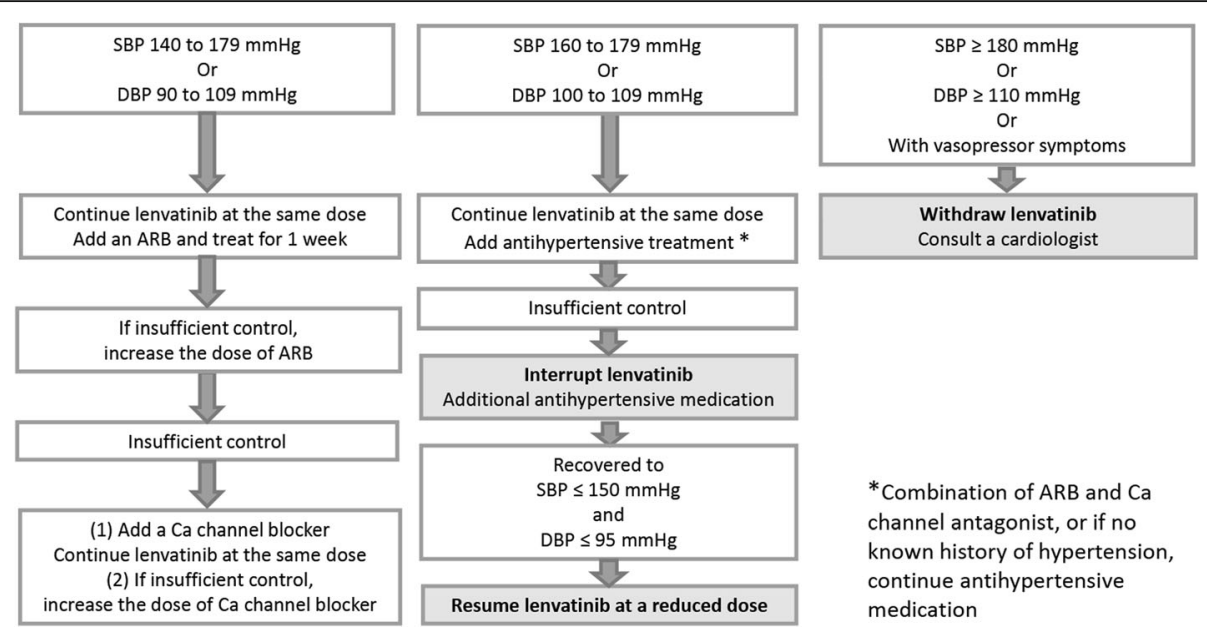

Fig. 1 Management of hypertension to enable continuation of lenvatinib treatment. The concomitant use of antihypertensive drugs and a change in the schedule of lenvatinib treatment should be considered according to the severity of hypertension. *Dose escalation is recommended up to a maximum dose for ARB or Ca channel blocker treatment. ${ }^{* *} \mathrm{~A}$ combination of ARB and Ca channel antagonist is recommended

at re-measurement, an antihypertension medication should be started. An antihypertensive drug should be initiated in patients with blood pressure $\geq 140 / 90 \mathrm{mmHg}$ or a diastolic blood pressure $>90 \mathrm{mmHg}$ in accordance with the guidelines for the management of hypertension [17-19]. Angiotensin II receptor blockers, angiotensinconverting enzyme inhibitors and calcium channel blockers are commonly used to treat MKI-induced hypertension. Although there is little evidence to suggest the superiority of one agent over another in this setting, we recommend the use of an angiotensin II receptor blocker or angiotensin-converting enzyme inhibitor as first-line treatment because of their renoprotective effect. If monotherapy is insufficient in lowering blood pressure, we consider dose escalation of the antihypertensive drug or initiating combination therapy with a calcium channel blocker.

If the systolic blood pressure is $\geq 160 \mathrm{mmHg}$ or diastolic blood pressure is $\geq 100 \mathrm{mmHg}$ despite antihypertensive therapy, lenvatinib treatment should be interrupted until a reduction in blood pressure is seen and lenvatinib can be then resumed at a reduced dose.

\section{Diarrhea}

Diarrhea is a manifestation of gastrointestinal toxicity and is common with all MKIs. The incidence of any grade of diarrhea was $60 \%$ in Japanese patients in the SELECT trial (Table 2) [7]. Diarrhea was generally mild, of grade 1 or 2 in severity. The median time to first onset was 12.1 weeks [20]. Supportive therapy is needed to prevent dehydration, and an antidiarrheal agent such as loperamide should be considered. Diphenoxylate and atropine (Lomotil) and budesonide are also useful treatments for diarrhea (not approved in Japan). Treatment interruption is required for grade 3 or 4 diarrhea and subsequent lenvatinib dose reductions may be necessary after resuming lenvatinib. In patients administered a diuretic for the management of hypertension, care should be taken to avoid dehydration.

\section{Fatigue}

Fatigue caused by suppression of thyroid function has been reported in clinical studies of other VEGFR inhibitors. Hypothyroidism has been reported in sorafenib-treated patients with renal cancer and in sunitinib-treated patients with gastrointestinal stromal cancer [21, 22]. Additionally, thyroid hormone replacement therapy improved fatigue in axitinib-treated patients with cancer [23]. Adjustment of the thyroid hormone dose is important in patients who experience fatigue, particularly in those with differentiated thyroid cancer for the maintenance of TSH suppression. Destructive thyroiditis and inhibition of VEGFR were proposed as possible mechanisms of thyroid dysfunction associated with tyrosine kinase inhibitors [24].

The incidence of fatigue in Japanese patients in the SELECT trial was relatively high, being $60 \%$ for any grade of fatigue and $13.3 \%$ for grade $\geq 3$ fatigue (Table 2 ) [7]. Onset of fatigue was seen early after initiation of lenvatinib, with a median time to first onset of 3 weeks [20]. Treatment interruption is recommended if patients experience intolerable fatigue. Severe fatigue can be managed by dose modification or discontinuation. Most patients recovered from fatigue within 1 week after interruption of lenvatinib.

\section{Palmar-plantar erythrodysesthesia}

Palmar-plantar erythrodysesthesia, known as hand-foot syndrome, is characterized by hyperkeratotic, tender 
lesions on areas of friction/pressure, and usually affects the palms and soles. The cutaneous lesions may be accompanied by paresthesia, tingling and burning sensations of the hands and feet. The incidence of palmarplantar erythrodysesthesia in Japanese patients in the SELECT trial was higher than that in the general population. The incidence for any grade was $70.0 \%$ in Japanese patients and $31.8 \%$ in the general population. However, the incidences of grade $\geq 3$ were similar (3.3\% in Japanese patients and $3.4 \%$ in the general population) $[2,7]$. The median time to first onset was 5.9 weeks [20].

The severity was grade 2 or less in most cases, enabling continuation of lenvatinib. Compared with the DECISION study of sorafenib, palmar-plantar erythrodysesthesia caused by lenvatinib is less likely to progress to grade $\geq 3$. There was no treatment discontinuation due to palmar-plantar erythrodysesthesia in the SELECT study; by contrast, several patients discontinued sorafenib because of palmar-plantar erythrodysesthesia [1].

Instructions for skin care of the hands and feet are needed at the initiation of lenvatinib treatment. Preventative measures should be taken, and the condition can be managed by application of topical $20 \%-40 \%$ urea cream and shaving the callouses in most cases (Fig. 2) [25]. A referral to a dermatologist and/or podiatrist may be necessary. If the condition is grade 1 in severity, lenvatinib treatment can be continued at the same dose with use of a moisturizing cream and a hydrocolloid dressing for the feet may be considered [26]. However, severe cases may require dose modification or treatment interruption. If the severity is grade 2, lenvatinib treatment should be interrupted and steroid ointment, such as difluprednate and betamethasone valerate, should be used, followed by consultation with a dermatologist.
After recovery to a lower grade, lenvatinib treatment may be resumed at the same dose. If grade 2 occurs within 1 week from initiation of lenvatinib treatment, or if grade 3 occurs in any cases, the incidence suggests that the patient's skin is sensitive to lenvatinib treatment. Treatment should be interrupted and resumed at the reduced dose after the recovery to grade 0 or 1 .

\section{Proteinuria}

In the SELECT trial, the incidence of proteinuria of any grade and grade $\geq 3$ was $63.3 \%$ and $20.0 \%$ in Japanese patients, and $31.0 \%$ and $10.0 \%$ in the general population, respectively (Table 2 ) [2, 7]. Proteinuria developed relatively early during lenvatinib therapy, with a median time to first onset of 6.1 weeks. The mechanism underlying proteinuria associated with VEGF inhibitors is unclear. One possible mechanism might be thrombotic microangiopathy, a condition that has been reported for other MKIs [27, 28]. This condition might impair VEGFR-expressing podocytes, which play a central role in glomerular filtration. In addition, because FGF is implicated in podocyte differentiation and recovery after injury, FGF receptor inhibition may adversely affect podocyte function in patients treated with a MKI [29]. However, the relationship between FGF receptor inhibition and toxicity remains unclear, and further investigations are needed.

Regular urinalysis should be conducted to detect the onset of proteinuria. Lenvatinib may be continued if proteinuria is grade 1 or 2 , based on the criteria in clinical trials $[7,10]$. However, if proteinuria is grade 3 or 4 (defined as urinary protein $\geq 3.5 \mathrm{~g} / \mathrm{d}$ or a urine proteinto-creatinine ratio $\geq 3.5$ ), or if grade 1 or 2 occurs in high-risk patients with edema, fluid collection or

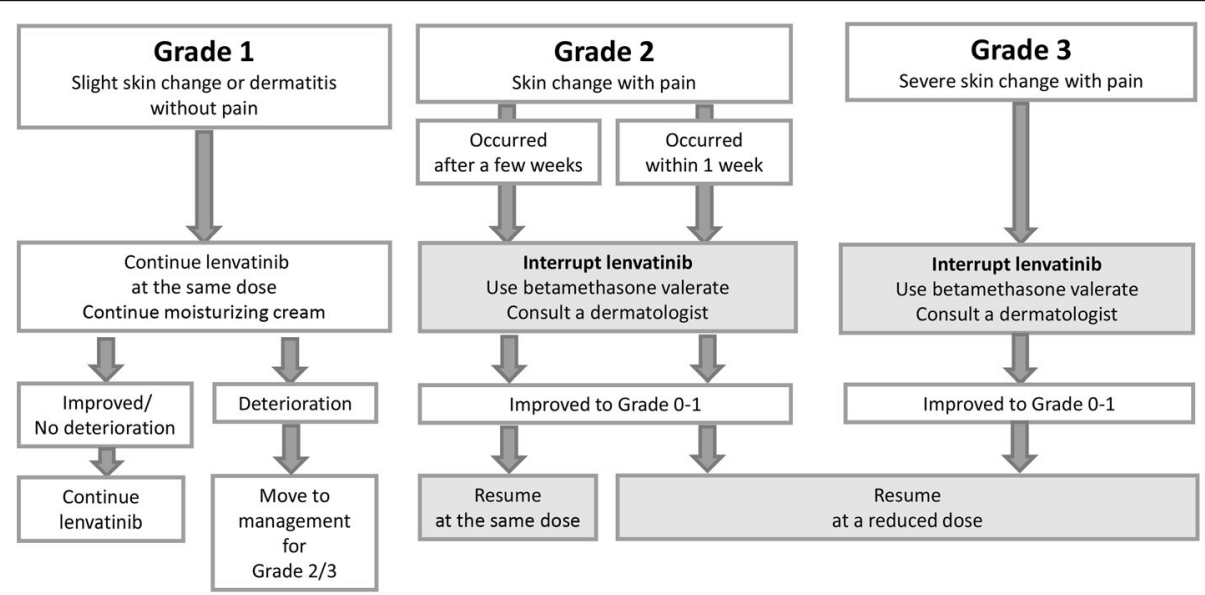

Fig. 2 Management of palmar-plantar erythrodysesthesia. Physicians should instruct patients to care for the skin on their hands and feet at the initiation of lenvatinib. The concomitant use of a moisturizing cream and steroid ointments, and a change in the schedule of lenvatinib treatment should be considered according to the severity of palmar-plantar erythrodysesthesia. *Steroid ointments of Group 2 (very strong: e.g., difluprednate) or Group 3 (strong: e.g., betamethasone valerate) are recommended. The treatment regimen and schedule should be determined by a dermatologist 
elevated serum creatinine, lenvatinib treatment should be interrupted and spot urine or 24-h urine should be checked to determine urinary protein and/or the urine protein-to-creatinine ratio. Lenvatinib may be continued at the same dose if urinary protein is $<3.5 \mathrm{~g} /$ day and there is no edema, fluid collection or elevation in serum creatinine. After the proteinuria has recovered or improved to a lower grade, lenvatinib treatment may be restarted at a reduced dose (Fig. 3).

Also, patients with renal dysfunction caused by diabetes or hypertension should be carefully managed. Prescriptions of diuretics for edema and a statin for hyperlipidemia are recommended. In addition, treatment interruption is also recommended if the patient experiences respiratory disorders (i.e., pulmonary edema or effusion), an increase in creatinine, or leg edema of any grade. Its administration may be resumed once these symptoms have improved. Resumption of lenvatinib at a reduced dose is usually recommended after interruption.

Irreversible decline of renal function associated with the VEGFR inhibitor sunitinib has been reported [30, 31], suggesting that VEGFR inhibitors should be discontinued prior to deterioration of proteinuria. Therefore, long-term monitoring for and management of proteinuria is required with lenvatinib treatment.

\section{Thrombocytopenia}

The incidence of thrombocytopenia in Japanese patients in the SELECT trial was $46.7 \%$ for any grade and $6.7 \%$ for grade $\geq 3$, indicating that most cases were of grade $\leq 2$, while the incidence in the overall population was $8.8 \%$ and $1.5 \%$, respectively (Table 2) [7]. The mechanism of thrombocytopenia in lenvatinib-treated patients is unknown, but it might involve inhibition of c-Kit and FGFR, both of which are involved in megakaryocytopoiesis [32]. Accordingly, inhibition of both pathways is expected to downregulate the production of megakaryocytes and platelets.

Thrombocytopenia increases the risk of bleeding especially in patients with grade 4 thrombocytopenia. Therefore, the platelet count should be carefully monitored. The dose of lenvatinib should be reduced or its administration interrupted in patients with grade 3 or 4 thrombocytopenia. Most cases of thrombocytopenia improved rapidly after drug interruption.

\section{Hemorrhage}

There is a risk of bleeding with all anti-angiogenic MKIs, and it most commonly manifests as epistaxis of mild severity. However, hemorrhage is a common cause of death in patients treated with anti-angiogenic MKIs [33]. Therefore, clinicians should be vigilant for this complication. Clinicians should also pay particular attention to the risk of hemorrhage in patients with thyroid carcinoma, as explained below.

In the SELECT trial, the incidence of any grade epistaxis in Japanese patients was $20 \%$, all of which were grades 1 or 2 [7]. Important information about the risk of bleeding in lenvatinib therapy has emerged from a post-marketing study in Japan (data on file, Eisai Co Ltd.; ClinicalTrials.gov Identifier: NCT02430714), in which 14 cases of hemorrhage were reported (Table 3). Notably, hemorrhage occurred in $3.4 \%$ (8 cases out of a total of 238 cases) of patients with anaplastic thyroid carcinoma, which is locally invasive, and in $0.8 \%$ (6 cases out of a total of 778 cases) of patients with differentiated thyroid cancer (Table 3). Most cases were associated with tumor shrinkage and necrosis, resulting in bleeding from the tumor or carotid artery (Table 3). The tumor had infiltrated the carotid artery or was located next to it in 12 cases. Therefore, there may be a risk of hemorrhage as a result of arterial invasion or dermal

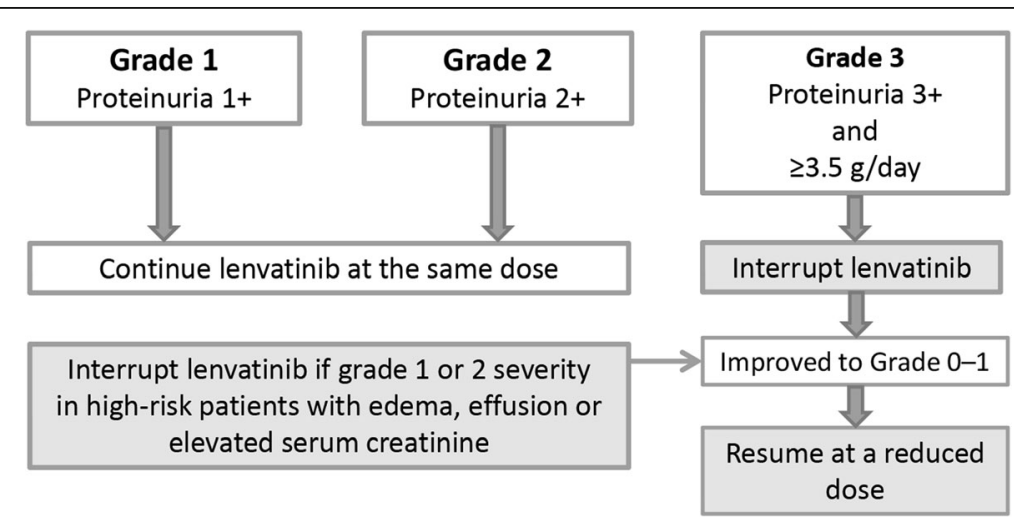

Fig. 3 Management of proteinuria to enable continuation of lenvatinib treatment. A change in the schedule of lenvatinib treatment should be considered according to the severity of proteinuria. Careful management of patients with renal dysfunction caused by diabetes or hypertension should be considered. Prescriptions of a diuretic for edema and a statin for hyperlipidemia are recommended 


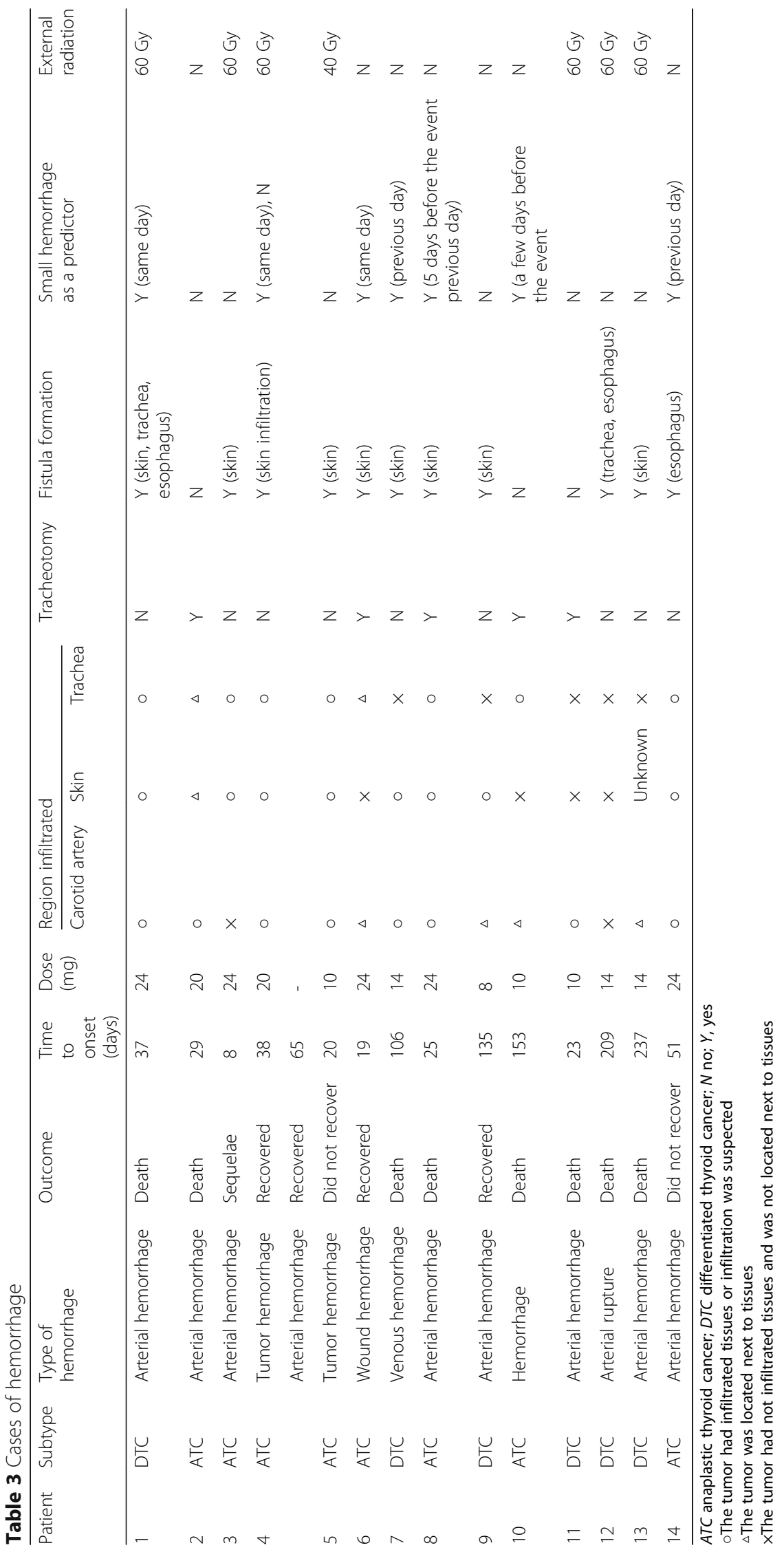


fistula formation, which can lead to serious complications and death.

Bleeding occurred within a few months after initiation of lenvatinib in more than half of the cases and may be due to tumor shrinkage, especially in patients with anaplastic thyroid carcinoma. Therefore, diagnostic imaging should be considered monthly for at least the first 3 months of lenvatinib to identify the presence of tumor. In patients with a rapidly growing tumor or metastasis close to the carotid artery, jugular vein or hilus, the administration of MKIs should be carefully evaluated to avoid the risk of hemorrhage [33, 34].

In the above-mentioned post-marketing study, fistula formation occurred in 11 cases. The occurrence of a small hemorrhage as a predictor was observed in seven cases. Seven cases received external radiation. It is uncertain whether radiation therapy should be prioritized over systemic therapy to achieve local disease control. The risk of skin fistula formation must be considered when lenvatinib is combined with radiation therapy. If lenvatinib is to be initiated after radiation therapy to achieve local control, we suggest that lenvatinib should be initiated with caution after radiation therapy to avoid the risk of aerodigestive fistula formation [35, 36]. Hemorrhage was also reported in the area corresponding to a previous radiation field during treatment with the MKI sunitinib in patients with nasopharyngeal carcinoma [33]. The authors of that report proposed that direct vascular invasion by the tumor increased the risk of serious bleeding. Taken together, these findings suggest that vascular-targeting MKIs have a high risk of bleeding and that caution should be taken for disease conditions and treatment history. Instead of MKIs, other systemic therapies such as BRAF-directed therapy for those with a BRAF mutation [37-40] and paclitaxel may be recommended for the patients with a high risk of bleeding.

\section{Long-term outcomes of lenvatinib treatment and importance of managing adverse events}

Until recently, there had been no evidence that MKIs prolonged overall survival. The updated results of the SELECT trial were reported at the 2015 European Cancer Congress and they revealed an improvement in overall survival among patients with radioiodine-refractory differentiated thyroid cancer after adjustment by the rank-preserving structural failure time model [41]. Furthermore, an update of the SELECT trial results reported at the 2016 ASCO Annual Meeting indicated that the median progression-free survival and the median duration of response were 19.4 months and 30 months, respectively [42]. These results suggest that long-term administration of lenvatinib may be beneficial in patients with radioiodine-refractory differentiated thyroid cancer, and that lenvatinib may extend survival in patients with thyroid cancer. However, adverse events are relatively common and must be managed appropriately in order to allow lenvatinib to be administered for a sufficient length of time.

In the SELECT study, liver function tests were performed at baseline, every 2 weeks for 2 months, and monthly thereafter. Renal function, electrolytes, and serum calcium were checked monthly. TSH levels were examined at baseline and monthly. The presence of proteinuria was examined at baseline and periodically during treatment (urine dipstick, if 2+, then 24-h urine collection for measurement of urinary protein and/or urinary protein-to-creatinine ratio). Blood pressure was monitored after 1 week, then every 2 weeks for 2 months, and monthly thereafter. Because blood pressure fluctuates from day to day, frequent management, including self-monitoring by patients if possible, is required, and patients in the SELECT study were also asked to monitor their blood pressure. To monitor disease progression, imaging was performed at 8- to 12week intervals in the SELECT study.

The approach taken to manage adverse events should consider the type and severity of event, and its potential impact on the patient's daily life activities [12]. We recommend administration at the same dose in patients with low-grade adverse events, having little impact on treatment continuation, because low-grade adverse events can generally be managed using appropriate supportive strategies and monitoring dependent upon the event types. Such strategies include administration of antihypertensive drugs for hypertension and emollient creams for palmar-plantar erythrodysesthesia. Highergrade adverse events, however, might require a reduction in the lenvatinib dose and/or temporary treatment interruption until the adverse event has been resolved or its severity declined to an acceptable grade (i.e., to grade 0 or 1). Discontinuation as a possible outcome should also be considered and might be necessary for some patients. However, if the administration of lenvatinib is interrupted temporarily and subsequently resumed once the adverse event has resolved, we recommend administration at a reduced dose upon resuming treatment. Resumption without dose modification may be an option if supportive therapy is available to control the symptoms.

\section{Summary}

Lenvatinib has shown efficacy in stabilizing progressive thyroid cancer, with emerging evidence of a possible benefit in overall survival. However, the use of lenvatinib is associated with adverse events and standard strategies to manage them have not been established. As discussed in this review, adverse events induced by lenvatinib treatment may be managed by strategies that have been employed for other MKIs. 
Approaches including dose reduction, treatment interruption, and supportive medications and treatment discontinuation should consider the type and severity of the event, and its potential impact on the patient's daily activities. By carefully monitoring and managing adverse events, many patients may be able to continue lenvatinib treatment at the appropriate dose.

The combination of lenvatinib with other targeted agents, such as mTOR inhibitors [43-45], is a promising area of investigation, while MEK inhibitors, BRAF inhibitors $[37,38]$ and immune-oncology agents [46] also show promise for treating radioiodine-refractory, differentiated thyroid cancer. Adverse events may arise with these new agents and combination therapy, and they may warrant a comprehensive and multidisciplinary approach as required in the management of adverse events of lenvatinib.

A thorough understanding of adverse events and their management by patients through communication with their physicians and other medical staff is a key factor in successful long-term treatment with lenvatinib. This in turn leads to improved treatment of cancer patients with other kinase inhibitors showing similar toxicity profiles.

\section{Conclusion}

It is our opinion that the optimal timing of treatment and the management of adverse events are important for the safe and efficacious use of lenvatinib. Accordingly, thorough understanding of adverse events and their management by patients through communication with their physicians is a key factor in successful long-term treatment with lenvatinib.

\section{Abbreviations \\ ARB: angiotensin receptor blockers; ASCO: American Society of Clinical Oncology; ATC: anaplastic thyroid cancer; Cl: confidence interval; DCR: disease control rate; DTC: differentiated thyroid cancer; FGFR: fibroblast growth factor receptor; HR: hazard ratio; MKI: multitargeted kinase inhibitors; MTC: medullary thyroid cancer; mTOR: mechanistic target of rapamycin; NE: not estimable; ORR: overall response rate; OS: overall survival; PFS: progression-free survival; PPE: palmar-plantar erythrodysesthesia; VEGFR: vascular endothelial growth factor receptor}

\section{Acknowledgements}

The authors thank William Ng, MD, PhD, of Edanz Medical Writing for medical writing services, which were funded by Eisai Co Ltd.

\section{Funding}

Analysis, and interpretation of data were conducted by the authors. The data presented in Table 3 were collected from a post-marketing study conducted by Eisai Co., Ltd. in Japan. Eisai Co Ltd. funded the use of a medical writing service, but not the development of the original drafts/concept for this review by the authors.

\section{Availability of data and materials}

All figures and tables are original and were made by authors for this review. They have not been published elsewhere.

\section{Authors' contributions}

All authors contributed to the original concept for this review and to preparation of the manuscript, and have read and approved the final manuscript.

\section{Ethics approval and consent to participate}

The data presented in Table 3 were collected from a post-marketing study in Japan and have not been published. The study was conducted in accordance with Good Post-marketing Study Practice (GPSP) of the Ministry of Health, Labour, and Welfare, Japan. Approval by the ethics committee of each institution and collection of informed consent from patients were not mandatory according to the GPSP guidelines.

\section{Consent for publication}

Not applicable.

\section{Competing interests}

Dr. Takahashi, Dr. Kiyota, and Dr. Tahara have all received lecture fees from Eisai Co Ltd.

\section{Publisher's Note}

Springer Nature remains neutral with regard to jurisdictional claims in published maps and institutional affiliations.

\section{Author details}

'Department of Medical Oncology, Cancer Institute Hospital, Japanese Foundation for Cancer Research, 3-8-31 Ariake, Koto-ku, Tokyo 135-8550, Japan. ${ }^{2}$ Department of Medical Oncology and Hematology, Kobe University Hospital, Kobe, Japan. ${ }^{3}$ Department of Head and Neck Medical Oncology, National Cancer Center Hospital East, Kashiwa, Japan.

Received: 1 March 2017 Accepted: 18 August 2017

Published online: 24 October 2017

\section{References}

1. Brose MS, Nutting CM, Jarzab B, et al. Sorafenib in radioactive iodinerefractory, locally advanced or metastatic differentiated thyroid cancer: a randomised, double-blind, phase 3 trial. Lancet. 2014;384:319-28.

2. Schlumberger $M$, Tahara $M$, Wirth $L$, et al. Lenvatinib versus placebo in radioiodine-refractory thyroid cancer. New Engl J Med. 2015;372:621-30.

3. Wells SA, Robinson BG, Gagel RF, et al. Vandetanib in patients with locally advanced or metastatic medullary thyroid cancer: a randomized, doubleblind phase III trial. J Clin Oncol. 2012:30:134-41.

4. Tohyama O, Matsui J, Kodama K, et al. Antitumor activity of levatinib (E7080): an angiogenesis inhibitor that targets multiple receptor tyrosine kinases in preclinical human thyroid cancer models. J Thyroid Res. 2014:2014:638747.

5. Matsui J, Funahashi Y, Uenaka T, et al. Multi-kinase inhibitor E7080 suppresses lymph node and lung metastases of human mammary breast cancer tumor MDA-MB-231 via inhibition of vascular endothelial growth factor-receptor (VEGF-R) 2 and VEGF-R3 kinase. Clin Cancer Res. 2008;14:5459-65.

6. Krajewska J, Kukulska A, Jarzab B. Efficacy of lenvatinib in treating thyroid cancer. Expert Opin Pharmacother. 2016:17:1683-91.

7. Kiyota N, Schlumberger M, Muro K, et al. Subgroup analysis of Japanese patients in a phase 3 study of lenvatinib in radioiodine-refractory differentiated thyroid cancer. Cancer Sci. 2015;106:1714-21.

8. Cabanillas ME, Schlumberger M, Jarzab B, et al. A phase 2 trial of lenvatinib (E7080) in advanced, progressive, radioiodine-refractory, differentiated thyroid cancer: a clinical outcomes and biomarker assessment. Cancer. 2015; 121:2749-56.

9. Schlumberger M, Jarzab B, Cabanillas ME, et al. A phase II trial of the multitargeted tyrosine kinase inhibitor Lenvatinib (E7080) in advanced medullary thyroid cancer. Clin Cancer Res. 2016;22:44-53.

10. Takahashi S, Kiyota N, Yamazaki T, et al. Phase II study of lenvatinib in patients with differentiated, medullary, and anaplastic thyroid cancer: Final analysis results. J Clin Oncol. 2016;34(suppl):abstract 6088. Available at: http://meetinglibrary.asco.org/content/164977-176

11. Tahara M, Kiyota N. Yamazaki T et al. Front Oncol: Lenvatinib for anaplastic thyroid cancer; 2017. (in press). 
12. Elisei $\mathrm{R}$, Schlumberger MJ, Muller SP, et al. Cabozantinib in progressive medullary thyroid cancer. J Clin Oncol. 2013;31:3639-46.

13. Brose MS, Frenette CT, Keefe SM, et al. Management of sorafenib-related adverse events: a clinician's perspective. Semin Oncol. 2014;41:S1-16.

14. Small HY, Montezano AC, Rios FJ, Savoia C, Touyz RM. Hypertension due to antiangiogenic cancer therapy with vascular endothelial growth factor inhibitors: understanding and managing a new syndrome. Can J Cardiol. 2014;30:534-43.

15. Wasserstrum $Y$, Kornowski R, Raanani P, Leader A, Pasvolsky O, lakobishvili Z. Hypertension in cancer patients treated with anti-angiogenic based regimens. Cardio-Oncology. 2015;1:6.

16. Wirth $\sqcup$, Tahara M, Robinson B, et al. Treatment-emergent hypertension and efficacy in the Phase 3 Study of (E7080) LEnvatinib in Differentiated Cancer of the Thyroid (SELECT). Ann Oncol. 2014;25(suppl_4):iv340-56. https://doi.org/10.1093/ annonc/mdu340. Available at: http://oncologypro.esmo.org/Meeting-Resources/ ESMO-2014/Treatment-emergent-hypertension-and-efficacy-in-the-Phase-3-Studyof-E7080-LEnvatinib-in-Differentiated-Cancer-of-the-Thyroid-SELECT.

17. Shimamoto K, Ando K, Fujita T, et al. The Japanese Society of Hypertension Guidelines for the Management of Hypertension (JSH 2014). Hypertens Res. 2014;37:253-392.

18. ESH/ESC Task Force for the Management of Arterial Hypertension.. 2013 Practice guidelines for the management of arterial hypertension of the European Society of Hypertension (ESH) and the European Society of Cardiology (ESC): ESH/ESC Task Force for the Management of Arterial Hypertension. J Hypertens. 2013;31:1925-38.

19. James PA, Oparil S, Carter BL, et al. 2014 evidence-based guideline for the management of high blood pressure in adults: report from the panel members appointed to the eighth joint National Committee (JNC 8). JAMA. 2014;311:507-20.

20. Haddad Rl, Schlumberger M2, Wirth $L 3$, et al. Incidence and timing of common adverse events in Lenvatinib-treated patients from the SELECT trial and their association with survival outcomes. Endocrine. 2017. https://doi. org/10.1007/s12020-017-1233-5. [Epub ahead of print].

21. Ravaud A, Schmidinger M. Clinical biomarkers of response in advanced renal cell carcinoma. Ann Oncol. 2013;24:2935-42.

22. Rutkowski $P$, Bylina E, Klimczak A, et al. The outcome and predictive factors of sunitinib therapy in advanced gastrointestinal stromal tumors (GIST) after imatinib failure - one institution study. BMC Cancer. 2012;12:107.

23. Fujiwara Y, Kiyota N, Chayahara N, et al. Management of axitinib (AG013736)-induced fatigue and thyroid dysfunction, and predictive biomarkers of axitinib exposure: results from phase I studies in Japanese patients. Investig New Drugs. 2012;30:1055-64.

24. Ahmadieh H, Salti I. Tyrosine Kinase Inhibitors Induced Thyroid Dysfunction: A Review of Its Incidence, Pathophysiology, Clinical Relevance, and Treatment, Article. Biomed Res Int, ID. 2013;725410. https://doi.org/10.1155/2013/725410.

25. Lacouture ME, Ciccolini K, Kloos RT, Agulnik M. Overview and Management of Dermatologic Events Associated with targeted therapies for medullary thyroid cancer. Thyroid. 2014;24(9):1329-40.

26. Shinohara $\mathrm{N}$, Nonomura $\mathrm{N}$, Eto $\mathrm{M}$, et al. A randomized multicenter phase II trial on the efficacy of a hydrocolloid dressing containing ceramide with low-friction external surface for hand-foot skin reaction caused by sorafenib in patients with renal cell carcinoma. Ann Oncol. 2014;25:472-6.

27. Uy AL, Simper NB, Champeaux AL, Perkins RM. Progressive bevacizumab-associated renal thrombotic microangiopathy. NDT Plus. 2009;2:36-9.

28. Bollée G, Patey N, Cazajous G, Robert C, Goujon JM, Fakhouri F, Bruneval P, Noël LH, Knebelmann B. Thrombotic microangiopathy secondary to VEGF pathway inhibition by sunitinib. Nephrol Dial Transplant. 2009;24:682-5.

29. Reiser J, Sever S, Faul C. Signal transduction in podocytes - spotlight on receptor tyrosine kinases. Nat Rev Nephrol. 2014;10:104-15.

30. Kawsar HI, Bansal S, Myles JL. Acute renal failure: a rare side effect of sunitinib therapy. Med Oncol. 2012;29:3594-6.

31. Takahashi D, Nagahama K, Tsuura Y, Tanaka H, Tamura T. Sunitinib-induced nephrotic syndrome and irreversible renal dysfunction. Clin Exp Nephrol. 2012;16:310-5

32. Avraham H, Price DJ. Regulation of megakaryocytopoiesis and platelet production by tyrosine kinases and tyrosine phosphatases. Methods. 1999:17:250-64.

33. Hui EP, Ma BB, King AD, et al. Hemorrhagic complications in a phase ॥ study of sunitinib in patients of nasopharyngeal carcinoma who has previously received high-dose radiation. Ann Oncol. 2011;22:1280-7.
34. Onoda N, Tokumoto M, Noda S, et al. [A case of recurrent anaplastic thyroid cancer treated by lenvatinib after successful long-term multimodal therapy]. Nihon Rinsho Geka Gakkai Zasshi (J Jpn Surg Assoc). 2016;77:291-5. In Japanese.

35. Blevins DP, Dadu R, Hu M, et al. Aerodigestive fistula formation as a rare side effect of antiangiogenic tyrosine kinase inhibitor therapy. Thyroid. 2014;24:918-22

36. Haugen BR, Alexander EK, Bible KC, et al. 2015 American Thyroid Association management guidelines for adult patients with thyroid nodules and differentiated thyroid cancer: the American Thyroid Association guidelines task force on thyroid nodules and differentiated thyroid cancer. Thyroid. 2016;26:1-133.

37. Brose MS, Cabanillas ME, Cohen EEW, et al. Vemurafenib in patients with BRAF V600E-positive metastatic or unresectable papillary thyroid cancer refractory to radioactive iodine: a non-randomised, multicentre, open-label, phase 2 trial. Lancet Oncol. 2016;17:1272-82.

38. Falchook GS, Millward M, Hong D, et al. BRAF inhibitor Dabrafenib in patients with metastatic BRAF-mutant thyroid cancer. Thyroid. 2015:25(1):71-7.

39. Subbiah V, Kreitman RJ, Wainberg ZA, et al. Efficacy of dabrafenib (D) and trametinib (T) in patients (pts) with BRAF V600E-mutated anaplastic thyroid cancer (ATC). J Clin Oncol. 2017;35(suppl):abstract 6023.

40. Shah MH, Wei L, Wirth $L$, et al. Results of randomized phase II trial of dabrafenib versus dabrafenib plus trametinib in BRAF-mutated papillary thyroid carcinoma. J Clin Oncol. 2017;35(suppl):abstract 6022.

41. Guo M, Sherman S, Wirth L, Schlumberger M, Dutcus C, Robinson B, Tahara M, Latimer N. Overall survival gain with lenvatinib vs. placebo in radioactive iodine refractory differentiated thyroid cancer (RR-DTC): An updated analysis. European Cancer Congress 2015. Abstract 2805. Available at: http://www.eccocongress.org/Vienna2015/Scientific-Programme/Abstractsearch?abstractid=21093.

42. Gianoukakis AG, Mathias EG, Dutcus C, Kalantari P, Yoon S. Response to lenvatinib treatment in patients with radioiodine-refractory differentiated thyroid cancer (RR-DTC): Updated results from SELECT. J Clin Oncol. 2016;34(suppl):abstract 6089. Available at: http://meetinglibrary.asco.org/ content/165048-176

43. Schneider TC, de wit D, Links TP, Van Erp NP, Van der Hoeven JJM, Gelderblom H, Roozen ICFM, Bos M, Corver WE, Van Wezel T, Smit JWA, Morreau H, Guchelaar H-J, Kapiteijn E. Everolimus in patients with advanced follicular-derived thyroid cancer: results of a phase II clinical trial. J Clin Endocrinol Metab 2017;102(2):698-707.

44. Schneider TC, de Wit D, Links TP, et al. Beneficial effects of the mTOR inhibitor Everolimus in patients with advanced medullary thyroid carcinoma: subgroup results of a phase II trial. Int J Endocrinol. 2015;348124

45. Lim SM, Chang H, Yoon MJ, et al. A multicenter, phase II trial of everolimus in locally advanced or metastatic thyroid cancer of all histologic subtypes. Ann Oncol. 2013;24(12):3089-93.

46. Mehnert JM, Varga A, Brose $M$, et al. Pembrolizumab for advanced papillary or follicular thyroid cancer: preliminary results from the phase $1 \mathrm{~b}$ KEYNOTE-028 study. J Clin Oncol. 2016;34(suppl):abstract 6091.

\section{Submit your next manuscript to BioMed Central and we will help you at every step:}

- We accept pre-submission inquiries

- Our selector tool helps you to find the most relevant journal

- We provide round the clock customer support

- Convenient online submission

- Thorough peer review

- Inclusion in PubMed and all major indexing services

- Maximum visibility for your research

Submit your manuscript at www.biomedcentral.com/submit 